https://doi.org/10.31713/m1019

\title{
INCREASING OIL RECOVERY APPLYING ELECTRIC STIMULATION ON THE FORMATION
}

\author{
Akramov B.Sh. \\ Branch of the Russian State University of Oil and Gas \\ (National Research University) named after I.M. Gubkin \\ in Tashkent city (Republic of Uzbekistan), $\mathrm{PhD}$, professor of the \\ Department of Development of Oil, Gas and Gas Condensate Fields, \\ Uzbekistan
}

\section{Umedov Sh.Kh.}

Tashkent State Technical University named after Islam Karimov, DSc, head of the Mining Electromechanics department, Uzbekistan

\section{Khaitov O.G.}

Tashkent State Technical University named after Islam Karimov, $\mathrm{PhD}$, associate professor, head of the Mining department, Uzbekistan

\section{Nuriddinov J.F.}

Institute of Geology and Exploration of Oil and Gas Fields, engineer, Uzbekistan

\section{Gafurov Sh.O.}

Tashkent State Technical University named after Islam Karimov, assistant of the Geology of Oil and Gas department, Uzbekistan

\section{Abstract}

The work is devoted to increasing the degree of depletion of reserves of longterm exploited hydrocarbon deposits on the basis of the obtained results of theoretical and experimental studies of the application of electrodynamic technologies for stimulating the formation and bottomhole formation zone.

The electrolysis of formation fluids, water, oil-bearing rocks, is accompanied by a mass transfer, primary and secondary chemical reactions, the formation of all kinds of salts, alkalis and acids, new organic substances and all kinds of surfactants. Not only the liquid is subjected to electrolysis, but also the oil and gas bearing rocks themselves (solid electrolyte). The magnetic and electrical forces arising during the electric treatment of reservoirs make it possible to effectively drain heterogeneous reservoirs and extract residual oil from non-working layers.

The work also carried out experiments to study the effect of the electric field on the surface tension coefficient at the oil-water interface. 
The circumstance of an abrupt change in the surface tension coefficient at the oil-water interface makes it possible in principle to create conditions in the reservoir that make it possible to slow down the cusping processes by applying an electric field of various magnitudes or, in other words, by regulating the amount of mass transfer.

In numerical terms, the oil recovery factor without electrophysical treatment was $52.94 \%$. Under electrophysical impact, the oil recovery factor was $94.12 \%$, i.e. equaled to almost complete extraction of oil from the sample. In the field, this figure, of course, will decrease by 2-3 times, but it remains quite high in comparison with other methods of increasing oil recovery.

Thus, the studies performed on samples in laboratory conditions indicate the possibility of using constant electric fields to increase oil recovery from depleted watered formations. Electrochemical treatment of the formation can significantly increase the displacement of oil from the formation. The increase in oil displacement reaches $15-20 \%$ and more.

With the help of water alone, $58 \%$ of the oil (of its total volume in the sand) was displaced from the sand, and under electric field with a voltage of $10 \mathrm{~V}$ and $20 \mathrm{~V}$, the total amount of displaced oil, respectively, increased to 67 and $83 \%$. Thus, the laboratory studies performed on the samples also indicate the possibility of using constant electric fields to increase oil recovery from depleted watered formations.

The carried out theoretical and experimental studies show the possibility of using the technology of electrochemical and electrothermochemical leaching of oilsaturated rocks to intensify oil production. The effectiveness of the recommended technology is especially noticeable in fields that have entered the final stage of development with a high water cut.

\section{Introduction}

Practice shows that the widespread use of traditional waterflooding technology (the main method of developing hydrocarbon deposits in Kazakhstan) does not provide effective recovery of residual reserves from low-permeability and highly watered formations. Therefore, increasing the oil recovery from producing fields applying effective methods of stimulation on wateroil-saturated formations is an important scientific and technical task facing the oilmen of the republic.

Almost all oil fields in Uzbekistan are at the late and final stages of development, which are characterized by a constant decrease in the level of oil production with a simultaneous increase in water cut (90\% and more on average).

In addition, most of the residual reserves of these fields are hardto-recover. They are concentrated in low-permeability reservoirs of small thickness with high-viscosity oil and anomalous properties, 
with variable gas content and significant sizes of oil-water zones. These factors have led to the low efficiency of traditional waterflooding and create favorable preconditions for the introduction of modern EOR.

In general, for fields that are in a late stage of development, an alternative option for curbing the decline in oil production and increasing oil recovery factor may be stimulating the hard-to-recover and residual oil reserves through the expanded use of workovers, modern EOR methods and bottomhole formation zone (BHZ) treatment.

In the conditions of highly watered formations of the fields of Uzbekneftegaz JSC, which are at a late stage of development and are characterized by a high degree of reserves depletion, along with squeeze job and BHZ treatment, it is necessary to more widely use other technologies, in particular, electric stimulation (ES) on the formation and $\mathrm{BHZ}$, which allows to increase oil recovery factor and reduce the cost of oil production.

The technology of ES on the formation and BHZ is based on the effects of changing the structure of the void space of a microheterogeneous medium and the spatial structures of filtration flows as a result of passing specially selected pulses of electric current through the productive formation. Localization of the current density in thin capillaries, that limit the rate of filtration in the medium, makes it possible to increase the cross-section of the capillaries, and thus increase the permeability in the BHZ. The use of the electrocapillary effect (change in surface tension at the phase interface under the influence of an electric field) makes it possible to shift the phase equilibrium in the medium and increase the relative permeability of oil with a simultaneous decrease in the relative permeability of water. As a result, due to a change in the spatial structure of filtration flows in the medium, the water cut of the wells can sharply decrease for a long period.

Therefore, the research focused on new energy-saving technologies that aim at reducing the residual oil reserves at a late stage of operation, especially from water flooded formations, is one of the urgent problems of the theory and practice of oil production. 


\section{Laboratory studies of electrophysical treatment}

To study the effect of ES on oil-saturated rocks, we used a setup (Fig. 1), which met the following requirements: the possibility of applying an electric field; when filtering water under the pressure gradient and an electric field, the inlet end of the sample should not become contaminated, and gas in the form of a bubble should not enter it; the sample should not be heated during the experiment.

The samples were saturated with water, the flow rate of water through the sample was determined at a constant pressure gradient in all experiments, and the permeability coefficient was calculated. On the next step, the flow rate of the liquid was determined with the electric field applied.

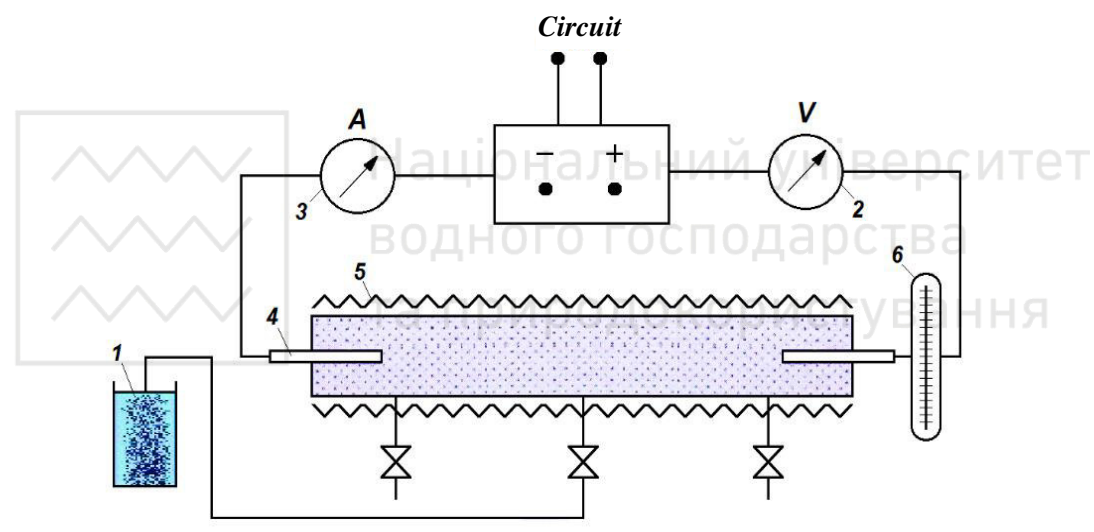

Fig. 1. Schematics of experimental setup

The studies were carried out with samples with a permeability coefficient of 2.2-0.73 D. To track the rate of advancement of the oilwater contact, an inductive control method was developed. The essence of the method lies in the fact that the dimensionless parameter of the quality factor of the inductor $Q=W Z / R$ (where $W$ is the frequency, $Z$ is the inductance, $R$ is the active resistance) depends on the electrical resistivity of the core, which is the core under study or the reservoir model.

It has been theoretically and experimentally proved that the resistivity of the most liquid-saturated rocks is proportional to the resistance of the liquid saturating them. By the change in the $Q$ - 
factors of sections of the coil in time, one can also estimate the completeness of the displacement of one agent by another.

According to the electroosmotic theory, when a liquid comes into contact with solid particles, due to the electrochemical interaction of molecules, solid particles are negatively charged, and an equal positive charge is distributed over the thickness of the adjacent liquid. The density of the volumetric row of liquid decreases with an increase in the distance to the surface of the particle at a distance $d$ and is characterized by a surface density $\delta$. Such a system is a capacitor, the capacity of which per unit area is $C_{0}=E E_{o} / d$, and has a charge $\delta$.

In the case of simultaneous electroosmotic and hydromechanical exposure, according to the principle of independence of action, the resulting fluid velocity will be equal to $v=v_{\mathrm{e}}+v_{\mathrm{o}}=k \operatorname{grad}\left(k_{2} \gamma+h\right)$. This equation is the main one for calculating filtration processes, both in the presence and in the absence of an electric field.

The experimental setup (Fig. 1) was used to study the effect of electroosmosis on oil recovery when oil is displaced by water. Samples were saturated with aqueous solutions and the permeability of the sample was determined. Water from the sample was displaced by oil from the XIX horizon of the Parsumurun dome of the Uzen field with the following characteristics: density $-0.785 \mathrm{~g} / \mathrm{cm}^{3}$, dynamic viscosity $-4.19 \mathrm{mPa} \cdot \mathrm{s}$, paraffin content $-21.8 \% \mathrm{wt}$.

The amount of displaced water was used to determine the amount of oil in the sample. Then the oil was displaced from the sample with water without electroosmotic action. When the recovery of oil stopped, the amount of displaced oil was measured. Further, the displacement of oil by water was carried out at the same pressure gradient, i.e. $10^{-3} \mathrm{MPa} / \mathrm{cm}$, but imposing electric field with a gradient of $10 \mathrm{~V} / \mathrm{cm}$. As a result, an additional amount of oil was displaced. The experiment was terminated when no more oil was releasing from the sample. In all experiments, the temperature at the outlet of the samples was maintained at a level of $20^{\circ} \mathrm{C}$.

In all cases, the use of electroosmotic treatment led to the release of an additional amount of oil from the sample, which obviously led to a decrease in the residual oil saturation. An increase in oil 
recovery was obtained within a few percent of the original oil volume.

Another series of experiments was carried out using kerosene as a hydrocarbon liquid. Experiments were carried out with several samples from sandstone containing 6\% montmorillonite (№ 1), 6\% pealite (№ 2) and 6\% kaolinite (№ 3). A control sample (№ 4) was made from pure sandstone. The magnitude of the current in the experiments varied from 5 to $15 \mathrm{~mA}$. From the graph (Fig. 2) it can be seen that the most noticeable application of the electric field affected the sample № 1 and quite insignificant - on the sample of pure sandstone (№ 4). It is interesting to note that after filtration, the voltage on the sample turned out to be 2 times higher than the initial one. This suggests that, apparently, irreversible changes have occurred in the structure of montmorillonite under the influence of an electric field. As a filtrating liquid in these experiments, a solution of $0.5 \mathrm{wt} \% \mathrm{NaCl}$ and $0.5 \%$ wt. $\mathrm{CaCl}$ was used.

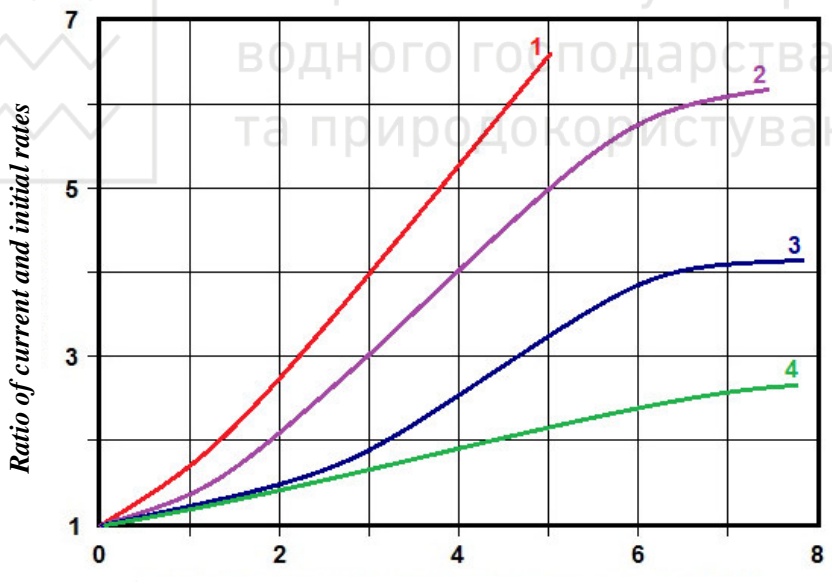

Gradient of the electric field potential, V/cm

Fig. 2. Increase in the rate of water filtration (the ratio of the current rate and the rate at the beginning of the experiment $q_{t} / q_{0}$ ) through sandstone samples when a direct current is applied: 1 - montmorillonite, $6 \%$; 2 - pealite, $6 \% ; 3$ - kaolinite, $6 \%$; 4 - sand, $100 \%$

Experiments on the joint flow of immiscible fluids were carried out on a sample of natural sandstone. The experiment was carried out as follows. A core sample was initially saturated with mineralized 
water. Then the mixture was filtered through the sample until a steady flow of both phases was observed. Then the voltage of a direct current was applied to the electrodes. After some time, the filtration rates of the liquids were measured again, and the temperature of the liquids leaving the sample was also recorded. The current was increased from 0.5 to $3.0 \mathrm{~mA}$.

In these experiments, it was found that under a constant current, the volumetric filtration rate of kerosene increased, and, consequently, increased the value of the effective permeability for kerosene depending on the voltage applied to the electrodes (Fig. 3).

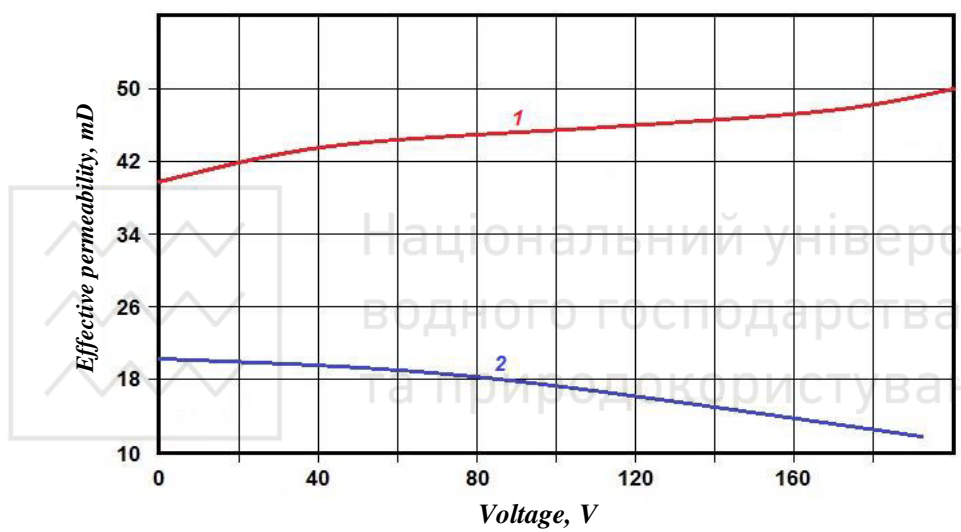

Fig. 3. Dependencies of effective permeability for kerosene (1) and water (2) from voltage

Also, experiments were carried out to study the effect of an electric field on the surface tension coefficient at the oil-water interface. In this case, a device was used in which the change in surface tension was recorded by the rate of oil flow from the capillary into the water at different voltages on the electrodes. It is established that the time between the separation of oil droplets from the capillary into the water without voltage supply practically does not change at $\mathrm{t}{ }^{\circ} \mathrm{C}=$ const. When a positive potential is imposed on oil, and a negative potential on water $(U=5 \mathrm{kV})$, the time slightly increases, i.e. the coefficient of surface tension at the oil-water interface increases. Then, after 1.5-2.5 minutes, $\delta$ decreases and remains at that level for indefinitely long. 
This abrupt change in the surface tension coefficient at the oilwater interface makes it possible to consider it fundamentally possible to create conditions in the reservoir that make it possible to slow down the processes of cusping by applying an electric field of various magnitudes or, in other words, to regulate the amount of mass transfer.

As it is known, the rate of mass transfer when extracting a liquid with a liquid is proportional to the mass transfer coefficient of the interface area (contact surface) and the driving force (pressure drop). With constant driving force, any attempt to increase the rate of mass transfer must, therefore, be aimed at increasing the other two factors. Also known the fact of a spontaneous increase in the coefficient of mass transfer due to the Maragoni effect which is a surface flow due to the appearance of a gradient of interfacial tension at the interface.

During waterflooding of oil deposits, the capillary forces manifest themselves variously. In a hydrophilic reservoir, at the front of water penetration into an oil reservoir, capillary forces contribute to the displacement of oil. In flooded zones and layers, capillary forces determine the residual oil saturation, which reduces the final recovery factor.

A direct consequence of capillary imbibition of layer-watered plays will be "mixing" of oil and water - an increase in oil saturation of the flooded layers, alignment of the phase saturation in the volume of the reservoir.

The depth of capillary imbibition naturally occurring in the formation can be determined by the formula

$$
h=\sqrt{\frac{\delta \cos \theta m^{0,5} t\left(k_{\alpha v \cdot 0}-k_{\alpha v \cdot w}^{0,5} k_{\alpha \cdot p}^{0,5}\right)}{0,00057 \eta_{w} \mu_{\alpha v} \tau k_{\alpha v}^{0,5}}}
$$

where $\delta$ is the coefficient of surface tension at the oil-water interface; $\theta$ - contact angle of wetting; $m$ - porosity; $t$ - exposure time; $k_{a v . o}$ - average oil permeability coefficient; $k_{a v . w}$ - average coefficient of water permeability; $k_{a v . p}$ - average pore permeability coefficient; $\eta_{w}$ - coefficient of oil displacement by water in flooded canals $\eta_{w}=\left(1-S_{o}-S_{o r}\right) /\left(1-S_{o}\right) ; \mu_{a v}$ - average viscosity of the liquid along the filtration path, $\mu_{a v}=\left(\mu_{o}+\mu_{w}\right) / 2 ; S_{o}$ - free oil saturation; $S_{o r}$ residual oil saturation; $\tau$ - tortuosity coefficient, $\tau=\chi / 1=2-2.5 ; \chi$ - the 
length of the path passed by the liquid; $l$ is the geometric length of the porous medium.

Calculations demonstrate that an increase in surface tension at the oil-water interface due to the effect of electrostatic forces insignificantly increases the depth of capillary imbibition and, thereby, the sweep factor. However, the variability of the surface tension over time radically changes this process, increasing the rate of the capillary imbibition due to a cyclic effect on the pore space of the reservoir.

As can be seen from Fig. 4, the additional sweep factor due to the electrical effect on $\delta$ with all constant residual parameters is 0.15 0.18 , which favorably affects the development parameters and, in particular, the oil recovery.

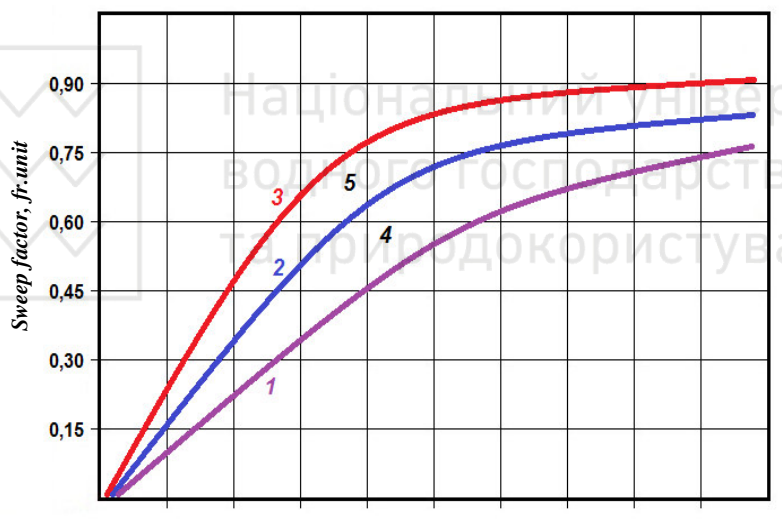

Time, $t$,

Fig. 4. Relative volume of fluid pumped through the reservoir: 1 - sweep factor without capillary imbibition; 2 - sweep factor taking into account capillary imbibition; 3 - sweep factor, taking into account capillary imbibition under the influence of an electric field; 4 - additional sweep factor due to capillary forces; 5 additional sweep factor due to electrical impact on the surface tension coefficient at the oil-water interface

In this paper, the analysis of the displacement characteristics was carried out using the Nazarov-Sipachev method, where the dependence of $V_{1} / V_{0}=f\left(V_{w}\right)$ is supposed to be used to determine the initial recoverable oil reserves, where $V_{1}, V_{0}, V_{w}$ is the accumulated production of liquid, oil and water, respectively. After a short period 
of recovery, this relationship will become $V_{l} / V_{0}=b+\alpha V_{w}$, where $\alpha$ is the slope of the straight line; $b$ is the segment cut off on the axis.

Transforming the equations, at $V_{w} \rightarrow \infty$, we obtain $V_{o} \rightarrow \alpha^{-1}$, thus, the inverse of the slope $\alpha$ of the straight line characterizes the value of the initial recoverable oil reserves.

As can be seen from Fig. 5, the final recovery factor when oil is displaced by water without ES is significantly less than when displacing with ES. In addition, the graphs show that the curves of the dependence of $K_{p} / K$ from water saturation after electric treatment shift to the right, thereby reducing the phase permeability.

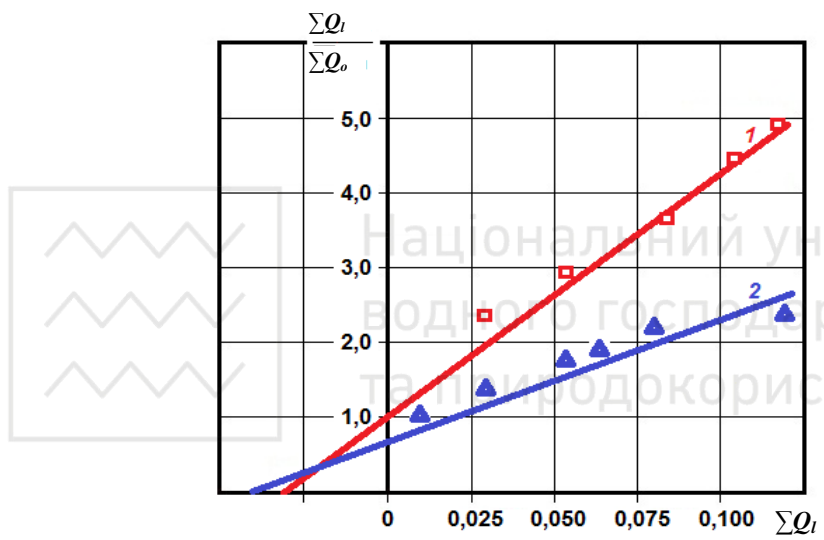

Fig. 5. Graph for the forecast of the final oil recovery: 1 - without electric stimulation; 2 - with electric stimulation

In numerical terms, the oil recovery factor without ES was $52.94 \%$. Under E, the oil recovery factor reached $94.12 \%$, i.e. almost complete extraction of oil from the sample. Thus, increased oil recovery amounted to $41.18 \%$. Under field conditions, this figure, naturally, will decrease by 2-3 times, but it remains quite high compared to other methods of increasing oil recovery.

Thus, the studies performed on samples under laboratory conditions indicate the possibility of using constant electric field to increase oil recovery from depleted watered formations. Electrochemical treatment of the formation can significantly increase the displacement of oil from the formation. The increase in oil displacement reaches $15-20 \%$ and more. The increase in oil 
displacement is caused by thermal and chemical effects of the current.

Studies on ES of reservoirs have shown that partially or heavily watered reservoirs are best suited for such treatment. The watered interlayers of oil reservoirs serve as conductors for current, and through them it is possible to act with heat and current on pure oil layers.

\section{Experimental studies of electrical treatment}

Experimental studies have been carried out below to study the features of electrolysis of a purely oil and water-oil (partially watered) reservoir and the effect of electric current on oil recovery (coefficient of oil displacement by water) from the reservoir. The experiments were carried out on reservoir models saturated with pure oil, water and oil. Oils used were from the Uzen field, and water aqueous solutions of salts $(\mathrm{NaCl})$ - formation and saline waters. The studies were carried out using direct current.

Based on the analysis of theoretical data on electrokinetic phenomena in capillary-porous media, we have developed several technological schemes for electrodynamic treatment of a formation in laboratory conditions, which make it possible to reveal the physical essence of electrokinetic phenomena in capillary-porous media.

To experimentally check the effectiveness of the effect of direct electric current on the change in the hydrodynamic permeability of the reservoirs, a device was used, with the help of which electroosmotic phenomena in the reservoirs were studied.

The studies were carried out using the UIK-5 unit on 2 sandstone samples taken from the well of the Uzen field.

Fig. 6 shows the results of the experiments carried out to determine $\eta=\left(V_{1}+V_{2}\right) / V_{2}$, where $V_{2}$ is the hydraulic filtration rate of a homogeneous liquid under the pressure drop at the ends of the sample; $V_{l}$ is the electroosmatic velocity of fluid transfer in the rock capillaries.

As follows from Figure 6, in all two studied samples during filtration of saline water with a concentration of $C=0.1$ and $\mathrm{NaCl}$ (1) and kerosene (2), with each pulse of increasing the gradient of the direct current potential in compliance with the polarity, a regular increase in the filtration rate is observed. Moreover, the filtration rate under the electric forces increases tens of times compared to the 
fixed filtration rate without voltage. Consequently, these results clearly show that with the help of constant electric field it is possible to intensify the inflow of fluid from the reservoirs.

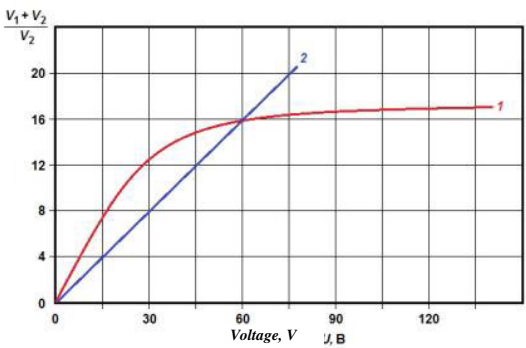

Fig. 6. Graphs of the dependence of $\eta$ from the voltage value $U .1$ - for saline water; 2 - kerosene

In addition, experiments on oil filtration through a capillary under electric field with the formation of an emulsion in a $10 \%$ aqueous solution of $\mathrm{NaCl}$ were carried out in a laboratory setup, and a special device was used to determine the oil recovery of sand under the influence of an electric field.

Fig. 7 shows a dependence of oil recovery of sand when it was flushed with water under the influence of electric field. As follows from the figure, $58 \%$ of the oil (of its total volume in the sand) was displaced from the sand using water alone. Under the action of an electric field with a voltage of $10 \mathrm{~V}$ and $20 \mathrm{~V}$, the total amount of displaced oil, increased to 67 and $83 \%$, respectively. Thus, the laboratory studies performed on the samples also indicate the possibility of using constant electric fields to increase oil recovery from depleted reservoirs producing under flooding.

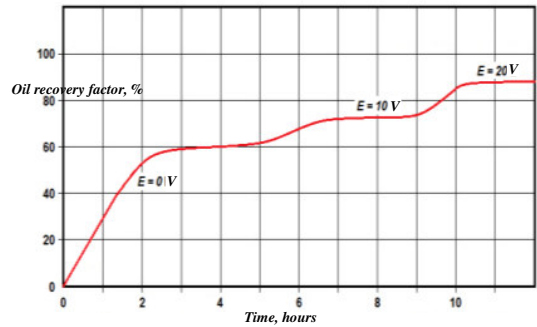

Fig. 7. Dependence of the oil displacement coefficient when flushing with water under the influence of an electric field 
Laboratory studies of the process of electrical treatment (electrolysis) of an oil reservoir in static conditions containing buried water were carried out using an oil-saturated linear reservoir model equipped with graphite electrodes at both ends and connected to a DC source (Figure 1). The experiments were carried out on reservoir models filled with sand $0.5-1 \mathrm{~mm}$ in diameter and saturated with oil, density of which was $870 \mathrm{~kg} / \mathrm{m}^{3}$. The water saturation of the reservoir is $30 \%$; buried water - formation water. Its mineralization $120 \mathrm{~g} / \mathrm{l}$. Formation porosity $30 \%$, permeability $200 \mathrm{mD}$, model length $75 \mathrm{~cm}$, diameter $3 \mathrm{~cm}$.

The results of the experiments led to the following conclusions:

- purely oil reservoirs, despite the presence of buried, saline water, have a relatively high resistivity. The resistivity of purely oil reservoirs fluctuates within 2-10 $\mathrm{Ohm} \cdot \mathrm{m}$. However, it is several orders of magnitude less than the resistivity of oil and minerals that make up the rock skeleton. Consequently, the conductivity of a purely oil reservoir is solely due to the conductivity of electrolyteburied saline water;

- at voltages of $50-220 \mathrm{~V}$ and more, the current was $20-50 \mathrm{~mA}$ due to the low conductivity of the formation, no noticeable heating of the formation during the period of the experiments was noted. The heating was mainly applied to the near-electrode zones.

The composition of the evolved gas in the near-electrode zones was determined according to the GOST 23781-87 method using a Kristall-5000 gas chromatograph (Russia). In this case, the cathode gas consists of homologues of methane and hydrogen, and the anode gas consists of homologues of methane, carbon dioxide, chlorine and oxygen.

To reveal the efficiency of electrical treatment of watered formations, we carried out experiments in glass formations up to 1.5 $\mathrm{m}$ long and $35-40 \mathrm{~mm}$ in diameter, filled with sand. At the same time, the permeability of the formations fluctuated within the range of 50-350 mD.

The pressure drop was determined from the condition of compliance with the parameters of the approximate simulation. In the experiments, oils with a viscosity of $15-20 \mathrm{mPa} \cdot \mathrm{s}$ were used. 
Analysis of the results of the experiments shows that in the experiments with electrical treatment, a significant (2-3 times) increase in the displacement of oil from the ionized formation was noticed. Studies on the electrical treatment of formations have shown that partially or heavily watered formations are best suited for ES. The watered interlayers of oil reservoirs serve as conductors for current, and through them it is possible to exert heat and current on pure oil layers.

Laboratory studies have shown that the electrolysis of formation water under dynamic conditions differs from the electrolysis of water under static conditions. To study this issue, we carried out special experiments on models of reservoirs filled with a porous medium and saturated with formation water.

The electrolysis was carried out at direct current at voltages of 75 and $120 \mathrm{~V}$ with periodic water supply and fluid withdrawal from the formation. Electrolysis was accompanied by gas formation, heating of the formation and the formation of alkaline solutions at the cathode, acid solutions at the anode. The resulting cathode solutions had a $\mathrm{pH}$ of more than 10 , anode $\mathrm{pH}$ of less than 1 .

\section{Conclusion}

1. Analysis of the performed theoretical and experimental studies shows the possibility of using the technology of electrochemical and electrothermochemical leaching of oil-saturated rocks to intensify oil production. The effectiveness of the recommended technology is especially noticeable in fields that have entered the final stage of development with a high water cut.

2. Experimental studies have established an increase in the rate of filtration of mineralized water and kerosene through sandstone samples, depending on the magnitude of voltage. The increase in the volume of displaced oil when exposed to electric current is $12-18 \%$ compared to that of when no voltage is applied.

3. To increase the displacing ability of the ES at the initial stage of field development, oil production should be carried out with simultaneous flooding of the oil-bearing formation, which will increase the conductivity of the electric current.

4. A calculation algorithm is proposed to determine the basic parameters of electric treatment of the formation for the resistance of 
2, 3 and 4 electrode borehole systems. The calculated data are in good agreement with the results of experimental studies.

5. An analytical expression for the dependence of the temperature distribution in the reservoir for one-, two- and multi-electrode systems has been obtained, which makes it possible to plot the temperature profile in the reservoir. The calculation results and the constructed temperature profiles fully confirm the terminal thermal effects arising in the near-electrode zones, which is the basis of the electro-thermal method of impact on the bottomhole zone.

6. Methods for the implementation of electrodynamic stimulation, which differ in the technology of their implementation, are proposed: electrodynamic method for cleaning the near-wellbore part of the formation; an electrochemical method for increasing oil recovery by influencing the formation with a direct electric current.

\section{References}

1. Akramov, B., Umedov, Sh., Nuriddinov, J., \& Mirsaatova, Sh. (2013). Electrophysical method of reservoir treatment as a method of intensifying oil production. Bulletin of TSTU, (4), 152-157.

2. Akramov, B., Khaitov, O., Nuriddinov, J., Gafurov, S., \& Joldasov, R. (2020). Development Indicators Forecasting Issues At The Chimion Deposit. Globus, (5(51)). doi:10.31618/2658-5197-2020-51-5-4

3. Akramov, B., Khaitov, O., \& Tabylganov, M. (2010). Methods for specifying initial and residual oil reserves based on development data at a later stage. Gorny Zhurnal, (2).

4. Akramov, B. S., Khaitov, O. G., \& Nuriddinov, J. F. (2017). Oil displacement by water in an electric field. Austrian Journal of Technical and Natural Sciences, 20-22. doi:10.20534/ajt-17-3.4-20-22

5. Akramov, B., Khaitov, O., Nuriddinov, J., \& Mirzakulova, M. (2019). Innovation in the development of fields with hard-to-recover reserves. International Scientific Forum "Science and Innovation - Modern Concepts", 1, 139-142.

6. Akramov, B., Khaitov, O., \& Nuriddinov, J. (2015). Oil displacement by water in an electric field. Europaische Fach-hochschule, 11, 38 